\title{
On a non-isothermal model for nematic liquid crystals
}

\author{
Eduard Feireisl* \\ Institute of Mathematics of the Czech Academy of Sciences \\ Žitná 25, 11567 Praha 1, Czech Republic \\ feireisl@math.cas.cz \\ Elisabetta Rocca ${ }^{\dagger}$ \\ Mathematical Department, University of Milan \\ Via Saldini 50, 20133 Milano, Italy \\ elisabetta.rocca@unimi.it \\ Giulio Schimperna \\ Mathematical Department, University of Pavia \\ Via Ferrata 1, 27100 Pavia, Italy \\ giusch04@unipv.it
}

May 24, 2022

\begin{abstract}
A model describing the evolution of a liquid crystal substance in the nematic phase is investigated in terms of three basic state variables: the absolute temperature $\vartheta$, the velocity field $\mathbf{u}$, and the director field $\boldsymbol{d}$, representing preferred orientation of molecules in a neighborhood of any point of a reference domain. The time evolution of the velocity field is governed by the incompressible NavierStokes system, with a non-isotropic stress tensor depending on the gradients of the velocity and of the director field $\boldsymbol{d}$, where the transport (viscosity) coefficients vary with temperature. The dynamics of $\boldsymbol{d}$ is described by means of a parabolic equation of Ginzburg-Landau type, with a suitable penalization term to relax the constraint $|\boldsymbol{d}|=1$. The system is supplemented by a heat equation, where the heat flux is given by a variant of Fourier's law, depending also on the director field $\boldsymbol{d}$. The proposed model is shown compatible with First and Second laws of thermodynamics, and the existence of global-in-time weak solutions for the resulting PDE system is established, without any essential restriction on the size of the data.
\end{abstract}

${ }^{*}$ The work of E.F. was supported by Grant 201/09/0917 of GA ČR in the framework of research programmes supported by AVČR Institutional Research Plan AV0Z10190503

$\dagger$ The work of E.R. was partially supported by the Nečas Center for Mathematical Modeling sponsored by MŠMT 


\section{Introduction}

Liquid crystals are materials with rheological properties between a conventional liquid and a solid crystal, where large elongate molecules give rise to a preferred orientation. Many different types of liquid crystals phases have been observed in practical experiments, distinguished by their characteristic optical properties (such as birefringence). When viewed under a microscope with a polarized light source, different liquid crystal phases will appear to have distinct textures. The contrasting areas in the textures correspond to domains where the liquid crystals molecules are oriented in different directions. Within a specific domain, however, the molecules are well ordered.

Theoretical studies of these types of materials are motivated by real-world applications. Proper functioning of many practical devices relies on optical properties of certain liquid crystalline substances in the presence or absence of an electric field. Typically, a liquid crystal layer sits between two polarizers that are crossed. The liquid crystal alignment is chosen so that its relaxed phase is twisted. This twisted phase reorients light passed through the first polarizer, allowing its transmission through the second polarizer (and reflecting back to the observer if a reflector is provided). The device thus appears transparent. When an electric field is applied to a liquid crystal layer, the long molecular axes tend to align parallel to the electric field thus gradually untwisting in the center of the liquid crystal layer. In such a state, the liquid crystals molecules do not reorient light, so the light polarized at the first polarizer is absorbed by the second polarizer, and the device loses transparency with increasing voltage. In this way, the electric field can be used to make a pixel switch between transparent or opaque on command. Color LCD systems use the same technique, with color filters used to generate red, green, and blue pixels. Similar principles can be used to make other liquid crystal based optical devices.

There have been numerous attempts to formulate continuum theories describing the behavior of liquid crystals flows. We refer to the seminal papers [4, 10], where Leslie and Ericksen provide a mathematical description of various properties of these materials, and to Lin and Liu [12] for the first attempt to analyze the model mathematically. We point out that, to the present state of knowledge, three main types of liquid crystals are distinguished, termed smectic, nematic and cholesteric. The smectic phase forms well-defined layers that can slide one over another in a manner very similar to that of a soap. The smectics are ordered along one direction. In the smectic A phase, the molecules are oriented along the layer normal, while in the smectic C phase, they are tilted away from the layer normal. These phases are liquid-like within the layers. There are many different smectic phases characterized by different types and degrees of positional and orientational order. The nematic phase appears to be the most common, where the molecules do not exhibit any positional order, but they have long-range orientational order. Thus, the molecules flow and their center of mass positions are randomly distributed similarly to a liquid, but they all point in the same direction (within each specific domain). Most nematics are uniaxial: they possess a preferred axis that is longer, with the other two being equivalent (so they can be approximately described as cylinders). Some liquid crystals are biaxial nematics, meaning that, in addition to orienting their long axis, they can also orient along a secondary axis. Crystals in the cholesteric phase exhibit a twisting of the molecules 
perpendicular to the director, with the molecular axis parallel to the director. The main difference between the nematic and cholesteric phases is that the former is invariant with respect to certain reflections while the latter is not.

In this paper, we consider the range of temperatures typical for the nematic phase. As already pointed out, the nematic liquid crystals are composed of rodlike molecules, with the long axes of neighboring molecules aligned. Such a kind of anisotropic structure may be described by means of a dimensionless unit vector $\boldsymbol{d}$, called director, that represents the direction of preferred orientation of molecules in a neighborhood of any point of a reference domain. In many experiments, the samples of nematic liquid crystals consist of slowly moving particles, therefore a relevant approach might be to study the behavior of director field $\boldsymbol{d}$ alone in the absence of velocity. However, the flow velocity $\mathbf{u}$ evidently disturbs the alignment of the molecules and also the converse is true: a change in the alignment will produce a perturbation of the velocity field $\mathbf{u}$. Hence, both $\boldsymbol{d}$ and $\mathbf{u}$ are relevant in the dynamics, and, to a certain extent, also the changes of the temperature $\vartheta$ (internal energy). We introduce a very simple non-isothermal model for nematic liquid crystals in the spirit of the simplified version of the Leslie-Ericksen model proposed by Lin and Liu [11], and subsequently studied in [7, 13 .

In the proposed model, the time evolution of the velocity field $\mathbf{u}$ is governed by the standard incompressible Navier-Stokes system, with a non-isotropic stress tensor depending on $\nabla_{x} \mathbf{u}, \nabla_{x} \boldsymbol{d}$, where the transport (viscosity) coefficients vary with temperature. The dynamics of $\boldsymbol{d}$ is described by means of a parabolic equation of Ginzburg-Landau type, with a suitable penalization term to relax the constraint $|\boldsymbol{d}|=1$. The system is supplemented by a heat equation, where the heat flux is given by a variant of Fourier's law in which the dependence on the director field $\boldsymbol{d}$ is taken into account, see Section 2. Although such a model may seem rather naive from the point of view of real-world applications, the present system of equations captures the essential mathematical features of the problem, and, last but not least, it is compatible with all underlying physical principles, in particular with First and Second laws of thermodynamics (as we shall see in Section 2.2).

Our goal is to show existence of global-in-time weak solutions to the system, without any essential restriction imposed on the size of initial data. In order to avoid problems caused by the interaction of the fluid with a kinematic boundary, we suppose the latter is impermeable and perfectly smooth imposing the complete slip boundary conditions on the velocity $\mathbf{u}$. The existence of weak solutions to the standard incompressible Navier-Stokes system was established in the celebrated paper by Leray [9]. One of the major open problems is to clarify whether or not the weak solutions also satisfy the corresponding total energy balance, more precisely, if the kinetic energy of the system dissipates at the rate given by the viscous stress. Even the so-called suitable weak solutions introduced by Caffarelli, Kohn and Nirenberg [3] allow for an uncontrolled dissipation of the kinetic energy that may not be captured by any term appearing in the classical formulation of the problem. Since the loss of kinetic energy in any energetically closed system must be compensated by a source term in the internal energy balance, the above mentioned problem causes unsurmountable mathematical difficulties whenever the equations for the kinetic and internal (heat) energy are sep- 
arated. To avoid this apparent difficulty, we use the idea proposed in [5] replacing the heat equation by the total energy balance. Of course, the price to pay is the explicit appearance of the pressure in the total energy balance that must be handled by refined arguments. Apart from the fact that the resulting system is mathematically tractable, such an approach seems much closer to the physical background of the problem, being an exact formulation of the First law of thermodynamics.

Let us finally mention that, with respect to [5], the main difficulty here consists in the proof of sufficiently strong estimates on the director field $\boldsymbol{d}$ in order to pass to the limit in the approximate problem. In particular, the celebrated Gagliardo-Nirenberg inequality is needed in order to control the strongly nonlinear terms containing $\nabla_{x} \boldsymbol{d}$ in both the momentum equation and the internal energy balance (cf. equations (2.12) and (2.13) below).

The organization of the paper is as follows. In Section 2, we derive the model following the standard physical principles. The weak formulation, together with the main result, are stated in Section 3. In Section 4, we derive the necessary a priori estimates and establish weak compactness of a family of weak solutions subject to $a$ priori bounds. Finally, in Section 5, we introduce a family of approximate problems, based on Galerkin-type approximations, and construct a weak solution of the system.

\section{Mathematical model}

Suppose that the fluid occupies a bounded spatial domain $\Omega \subset \mathbb{R}^{3}$, with a sufficiently regular boundary. Let $\varrho=\varrho(t, x)$ and $\mathbf{u}=\mathbf{u}(t, x)$ denote respectively the mass density and the velocity in the Eulerian reference system. Accordingly, the mass conservation is expressed by means of continuity equation

$$
\partial_{t} \varrho+\operatorname{div}(\varrho \mathbf{u})=0,
$$

where, in addition, the standard incompressibility constraint

$$
\operatorname{div} \mathbf{u}=0
$$

is relevant in the context of nematic liquid crystals.

By virtue of Newton's second law, the balance of momentum reads

$$
\partial_{t}(\varrho \mathbf{u})+\operatorname{div}(\varrho \mathbf{u} \otimes \mathbf{u})=\operatorname{div} \mathbb{T}+\varrho \mathbf{f}
$$

where $\mathbb{T}$ is the Cauchy stress, and $\mathbf{f}$ is a given external force.

Motivated by Lin and Liu [11] we consider the stress tensor in the form

$$
\mathbb{T}=\mathbb{S}-\varrho \lambda(\vartheta)\left(\nabla_{x} \boldsymbol{d} \odot \nabla_{x} \boldsymbol{d}\right)-p \mathbb{I},
$$

where $p$ denotes the pressure, and $\mathbb{S}$ is the conventional Newtonian viscous stress tensor,

$$
\mathbb{S}\left(\vartheta, \nabla_{x} \mathbf{u}\right)=\mu(\vartheta)\left(\nabla_{x} \mathbf{u}+\nabla_{x}^{t} \mathbf{u}\right) .
$$

Note that the transport coefficients $\mu$ and $\lambda$ are functions of the absolute temperature $\vartheta$, see also a strongly related model by Blesgen [1]. More specifically, $\mu$ is the viscosity 
coefficient assumed always positive, while $\lambda$ denotes the thermal dilatation coefficient that is an increasing function of $\vartheta$.

We assume that the driving force governing the dynamics of the director $\boldsymbol{d}$ is of "gradient type" $\partial_{\boldsymbol{d}} J$, where the potential $J$ is given by

$$
J(\vartheta, \varrho, \boldsymbol{d})=W(\boldsymbol{d})+\frac{1}{\vartheta} G(\vartheta, \varrho) .
$$

Here $G$ is a regular function of $\vartheta$ and $\varrho$, and $W$ penalizes the deviation of the length $|\boldsymbol{d}|$ from the value 1 . $W$ may be a general function that can be written as a sum of a convex (possibly non smooth) part, and a smooth, but possibly non-convex one. A typical example is $W(\boldsymbol{d})=\left(|\boldsymbol{d}|^{2}-1\right)^{2}$. Consequently, $\boldsymbol{d}$ satisfies the following equation

$$
\partial_{t} \boldsymbol{d}+\mathbf{u} \cdot \nabla_{x} \boldsymbol{d}+\partial_{\boldsymbol{d}} W(\boldsymbol{d})=\frac{1}{\varrho} \operatorname{div}\left(\varrho \nabla_{x} \boldsymbol{d}\right) .
$$

Finally, in accordance with the First law of thermodynamics, the internal energy balance reads

$$
\partial_{t}\left(\varrho e_{\text {int }}\right)+\operatorname{div}\left(\varrho e_{\text {int }} \mathbf{u}\right)+\operatorname{div} \mathbf{q}=\mathbb{T}: \nabla_{x} \mathbf{u},
$$

where $e_{\text {int }}$ denotes the internal energy density and q its flux. Following Ericksen's model [4], the flux can be taken in the form

$$
\mathbf{q}=-\kappa(\vartheta) \nabla_{x} \vartheta-\left(\kappa_{\|}-\kappa_{\perp}\right)(\vartheta) \boldsymbol{d}\left(\boldsymbol{d} \cdot \nabla_{x} \vartheta\right),
$$

where $\kappa, \kappa_{\|}-\kappa_{\perp}$ are positive functions of the temperature. Finally, we take $e_{\text {int }}=c_{v} \vartheta$, where $c_{v}>0$ is the specific heat at constant volume.

Scaling the last equation to have $c_{v}=1$, we arrive at the following system:

$$
\begin{aligned}
& \partial_{t} \varrho+\operatorname{div}(\varrho \mathbf{u})=0, \\
& \operatorname{div} \mathbf{u}=0 \\
& \partial_{t}(\varrho \mathbf{u})+\operatorname{div}(\varrho \mathbf{u} \otimes \mathbf{u})+\nabla p=\operatorname{div} \mathbb{S}-\operatorname{div}\left(\varrho \lambda(\vartheta)\left(\nabla_{x} \boldsymbol{d} \odot \nabla_{x} \boldsymbol{d}\right)\right)+\varrho \mathbf{f}, \\
& \partial_{t}(\varrho \vartheta)+\operatorname{div}(\varrho \vartheta \mathbf{u})+\operatorname{div} \mathbf{q}=\mathbb{S}: \nabla_{x} \mathbf{u}-\varrho \lambda(\vartheta)\left(\nabla_{x} \boldsymbol{d} \odot \nabla_{x} \boldsymbol{d}\right): \nabla_{x} \mathbf{u}, \\
& \partial_{t} \boldsymbol{d}+\mathbf{u} \cdot \nabla_{x} \boldsymbol{d}+\partial W(\boldsymbol{d})=\frac{1}{\varrho} \operatorname{div}\left(\varrho \nabla_{x} \boldsymbol{d}\right) .
\end{aligned}
$$

\subsection{Boundary conditions}

Equations (2.10-2.14) must be supplemented by a suitable set of boundary conditions. In order to avoid the occurrence of boundary layers, we suppose complete slip boundary conditions for the velocity

$$
\left.\mathbf{u} \cdot \mathbf{n}\right|_{\partial \Omega}=0, \quad[\mathbf{T} \mathbf{n}] \times\left.\mathbf{n}\right|_{\partial \Omega}=0
$$

together with the no-flux boundary condition for the temperature

$$
\left.\mathbf{q} \cdot \mathbf{n}\right|_{\partial \Omega}=0
$$

and the Neumann boundary condition for the director field

$$
\left.\nabla_{x} d_{i} \cdot \mathbf{n}\right|_{\partial \Omega}=0 \text { for } i=1,2,3 .
$$


The last relation accounts for the fact that there is no contribution to the surface force $\mathbb{T} \mathbf{n}$ from the director $\boldsymbol{d}$. This type of boundary conditions not only simplifies the analysis but it is also suitable for implementation of a numerical scheme (cf. [13] for further comments on this topic).

\subsection{Energy, entropy}

Multiplying momentum equation (2.12) by $\mathbf{u}$ and adding the resulting expression to (2.13) we deduce the total energy balance in the form

$$
\begin{gathered}
\partial_{t}\left(\varrho\left(\frac{1}{2}|\mathbf{u}|^{2}+\vartheta\right)\right)+\operatorname{div}\left(\varrho\left(\frac{1}{2}|\mathbf{u}|^{2}+\vartheta\right) \mathbf{u}\right)+\operatorname{div}(p \mathbf{u})+\operatorname{div} \mathbf{q} \\
=\operatorname{div}(\mathbb{S u})-\operatorname{div}\left(\varrho \lambda(\vartheta)\left(\nabla_{x} \boldsymbol{d} \odot \nabla_{x} \boldsymbol{d}\right) \mathbf{u}\right)+\varrho \mathbf{f} \cdot \mathbf{u} .
\end{gathered}
$$

Moreover, using the boundary conditions (2.15), (2.16) we may integrate (2.18) over $\Omega$ to obtain

$$
\partial_{t} \int_{\Omega}\left(\frac{1}{2} \varrho|\mathbf{u}|^{2}+\varrho \vartheta\right)=\int_{\Omega} \varrho \mathbf{f} \cdot \mathbf{u}
$$

in particular, the total energy is a constant of motion as soon as $\mathbf{f} \equiv 0$.

Let us denote by $\Lambda(\vartheta)$ a primitive of $1 / \lambda(\vartheta)$. Testing (2.13) by $1 / \lambda(\vartheta)$ and (2.14) by $\left(\operatorname{div}\left(\varrho \nabla_{x} \boldsymbol{d}\right)-\varrho W^{\prime}(\boldsymbol{d})\right)$, integrating the sum of the resulting equations over $\Omega$, and using the boundary conditions (2.17), together with the equation of continuity (2.10), we get

$$
\begin{aligned}
& \int_{\Omega}\left(\partial_{t} \boldsymbol{d}+\mathbf{u} \cdot \nabla_{x} \boldsymbol{d}\right)\left(\operatorname{div}\left(\varrho \nabla_{x} \boldsymbol{d}\right)-\varrho \partial W(\boldsymbol{d})\right)+\partial_{t} \int_{\Omega}(\varrho \Lambda(\vartheta))+\int_{\Omega} \mathbf{q} \cdot \nabla_{x} \vartheta \frac{\lambda^{\prime}(\vartheta)}{(\lambda(\vartheta))^{2}} \\
& =\int_{\Omega} \frac{1}{\varrho}\left|\operatorname{div}\left(\varrho \nabla_{x} \boldsymbol{d}\right)-\varrho \partial W(\boldsymbol{d})\right|^{2}+\int_{\Omega} \frac{1}{\lambda(\vartheta)} \mathbb{S}: \nabla_{x} \mathbf{u}-\int_{\Omega} \varrho\left(\nabla_{x} \boldsymbol{d} \odot \nabla_{x} \boldsymbol{d}\right): \nabla_{x} \mathbf{u}
\end{aligned}
$$

and

$$
\begin{aligned}
& \int_{\Omega}\left(\partial_{t} \boldsymbol{d}+\mathbf{u} \cdot \nabla_{x} \boldsymbol{d}\right)\left(\operatorname{div}\left(\varrho \nabla_{x} \boldsymbol{d}\right)-\varrho \partial W(\boldsymbol{d})\right) \\
& =\partial_{t} \int_{\Omega}\left(-\varrho \frac{\left|\nabla_{x} \boldsymbol{d}\right|^{2}}{2}-\varrho W(\boldsymbol{d})\right)-\int_{\Omega} \varrho\left(\nabla_{x} \boldsymbol{d} \odot \nabla_{x} \boldsymbol{d}\right): \nabla_{x} \mathbf{u} .
\end{aligned}
$$

Thus, finally, we arrive at

where the quantity

$$
\begin{aligned}
\partial_{t} \int_{\Omega} \varrho(\Lambda(\vartheta)- & \left.\frac{\left|\nabla_{x} \boldsymbol{d}\right|^{2}}{2}-\varrho W(\boldsymbol{d})\right)=\int_{\Omega} \frac{1}{\varrho}\left|\operatorname{div}\left(\varrho \nabla_{x} \boldsymbol{d}\right)-\varrho \partial W(\boldsymbol{d})\right|^{2} \\
& +\int_{\Omega} \frac{1}{\lambda(\vartheta)} \mathbb{S}: \nabla_{x} \mathbf{u}-\int_{\Omega} \mathbf{q} \cdot \nabla_{x} \vartheta \frac{\lambda^{\prime}(\vartheta)}{(\lambda(\vartheta))^{2}}
\end{aligned}
$$

is the entropy density of the system. Accordingly, the expression under the integral signs on the right-hand side of (2.21) represents the entropy production. By virtue of the Second law of thermodynamics, the entropy production is non-negative for any physically admissible process, in particular, we need $\lambda^{\prime} \geq 0$. 


\section{Main results}

For the sake of simplicity, we restrict ourselves to the case of constant density, say $\varrho \equiv 1$, and $\mathbf{f} \equiv 0$. Accordingly, the problem (2.11 2.14), supplemented with the boundary conditions (2.15), (2.16), and the initial conditions

$$
\mathbf{u}(0, \cdot)=\mathbf{u}_{0}, \mathbf{d}(0, \cdot)=\mathbf{d}_{0}, \vartheta(0, \cdot)=\vartheta_{0},
$$

reads as follows

$$
\begin{aligned}
& \operatorname{div} \mathbf{u}=0, \\
& \partial_{t} \mathbf{u}+\operatorname{div}(\mathbf{u} \otimes \mathbf{u})+\nabla p=\operatorname{div} \mathbb{S}-\operatorname{div}\left(\lambda(\vartheta)\left(\nabla_{x} \boldsymbol{d} \odot \nabla_{x} \boldsymbol{d}\right)\right), \\
& \partial_{t} \vartheta+\operatorname{div}(\vartheta \mathbf{u})+\operatorname{div} \mathbf{q}=\mathbb{S}: \nabla_{x} \mathbf{u}-\lambda(\vartheta)\left(\nabla_{x} \boldsymbol{d} \odot \nabla_{x} \boldsymbol{d}\right): \nabla_{x} \mathbf{u}, \\
& \partial_{t} \boldsymbol{d}+\mathbf{u} \cdot \nabla_{x} \boldsymbol{d}+\partial W(\boldsymbol{d})=\Delta \boldsymbol{d},
\end{aligned}
$$

coupled with the boundary conditions (2.15 2.17) and the initial conditions (3.1).

To begin, we introduce a weak formulation of (3.2 3.5) and formulate our main result on the existence of global-in-time weak solutions, without any restriction imposed on the initial data.

\subsection{Weak formulation}

In the weak formulation, momentum equation (2.3), with the incompressibility constraint (2.2), and the boundary conditions (2.15), are replaced by a family of integral identities

$$
\int_{\Omega} \mathbf{u}(t, \cdot) \cdot \nabla_{x} \varphi=0 \text { for a.a. } t \in(0, T)
$$

for any test function $\varphi \in C^{\infty}(\bar{\Omega})$,

$$
\int_{0}^{T} \int_{\Omega}\left(\mathbf{u} \cdot \partial_{t} \varphi+\mathbf{u} \otimes \mathbf{u}: \nabla_{x} \varphi\right)=\int_{0}^{T} \int_{\Omega} \mathbb{T}: \nabla_{x} \varphi-\int_{\Omega} \mathbf{u}_{0} \cdot \varphi(0, \cdot)
$$

for any $\varphi \in C_{0}^{\infty}\left([0, T) \times \bar{\Omega} ; \mathbb{R}^{3}\right),\left.\varphi \cdot \mathbf{n}\right|_{\partial \Omega}=0$, where the Cauchy stress tensor $\mathbb{T}$ is related to the unknowns through the constitutive equation (2.4).

Equation (2.7) holds in the strong sense, thanks to the regularity obtained for $\boldsymbol{d}$. More specifically, we have

$$
\partial_{t} \boldsymbol{d}+\mathbf{u} \cdot \nabla_{x} \boldsymbol{d}+\partial W(\boldsymbol{d})=\Delta \boldsymbol{d} \text { a.e. in }(0, T) \times \Omega, \quad \nabla_{x} \boldsymbol{d}_{i} \cdot \mathbf{n}_{\mid \partial \Omega}=0, i=1,2,3 .
$$

In the weak formulation the total energy balance (2.18) is replaced by

$$
\begin{gathered}
\int_{0}^{T} \int_{\Omega}\left(\left(\frac{1}{2}|\mathbf{u}|^{2}+\vartheta\right) \partial_{t} \varphi+\left(\frac{1}{2}|\mathbf{u}|^{2}+\vartheta\right) \mathbf{u} \cdot \nabla_{x} \varphi+\mathbf{q} \cdot \nabla_{x} \varphi\right) \\
=\int_{0}^{T} \int_{\Omega} \mathbb{T} \mathbf{u} \cdot \nabla_{x} \varphi-\int_{\Omega}\left(\frac{1}{2}\left|\mathbf{u}_{0}\right|^{2}+\vartheta_{0}\right) \varphi(0, \cdot),
\end{gathered}
$$

for any $\varphi \in C_{0}^{\infty}([0, T) \times \bar{\Omega})$, where $\mathbb{T}$, q obey (2.4), (2.9) , and by the entropy inequality

$$
\partial_{t} \vartheta+\operatorname{div}(\vartheta \mathbf{u})+\operatorname{div} \mathbf{q} \geq \mathbb{S}: \nabla_{x} \mathbf{u}-\lambda(\vartheta)\left(\nabla_{x} \mathbf{d} \odot \nabla_{x} \mathbf{d}\right): \nabla_{x} \mathbf{u} \quad \text { in } \mathcal{D}^{\prime}((0, T) \times \Omega),
$$

with $\mathbf{q}$ as in (2.9) and $\mathbb{S}$ as in (2.5). A weak solution is a triple $(\mathbf{u}, \boldsymbol{d}, \vartheta)$ satisfying (3.6 3.10). 


\subsection{Main existence theorem}

Before formulating the main result of this paper, let us state the list of hypotheses imposed on the constitutive functions. We assume that

$$
W \in C^{2}\left(\mathbb{R}^{3}\right), \quad W \geq 0, \quad \partial W(\mathbf{d}) \cdot \mathbf{d} \geq 0 \text { for all }|\mathbf{d}| \geq D_{0}
$$

for a certain $D_{0}>0$.

In addition, the transport coefficients are continuously differentiable functions of the absolute temperature satisfying

$$
0<\underline{\mu} \leq \mu(\vartheta) \leq \bar{\mu}, \quad 0<\underline{\kappa} \leq \kappa(\vartheta),\left(\kappa_{\|}-\kappa_{\perp}\right)(\vartheta) \leq \bar{\kappa} \text { for all } \vartheta \geq 0
$$

for suitable constants $\underline{\kappa}, \bar{\kappa}, \mu, \bar{\mu}$.

Finally, let $\lambda \in C^{1}([0, \overline{+}+))$ be such that

$$
\lambda^{\prime}(\vartheta) \geq 0, \quad \lambda^{\prime}(0)>0, \quad \lambda(0)=0, \quad \lambda(\vartheta) \leq \bar{\lambda} \text { for all } \vartheta \geq 0
$$

for a certain $\bar{\lambda}>0$.

Our main result reads as follows.

Theorem 3.1. Let $\Omega \subset \mathbb{R}^{3}$ be a bounded domain of class $C^{2+\nu}$ for some $\nu>0$. Assume that hypotheses (3.11-3.13) are satisfied. Finally, let the initial data be such that

$$
\begin{gathered}
\mathbf{u}_{0} \in L^{2}\left(\Omega ; \mathbb{R}^{3}\right), \operatorname{div} \mathbf{u}_{0}=0, \mathbf{d}_{0} \in L^{\infty} \cap W^{1,2}\left(\Omega ; \mathbb{R}^{3}\right), \\
\vartheta_{0} \in L^{1}(\Omega), \operatorname{ess}_{\inf } \vartheta_{0}>0 .
\end{gathered}
$$

Then problem (3.6 3.10) possesses a weak solution $(\mathbf{u}, \mathbf{d}, \vartheta)$ in $(0, T) \times \Omega$ belonging to the class

$$
\begin{gathered}
\mathbf{u} \in L^{\infty}\left(0, T ; L^{2}\left(\Omega ; \mathbb{R}^{3}\right)\right) \cap L^{2}\left(0, T ; W^{1,2}(\Omega)\right), \\
\mathbf{d} \in L^{\infty}\left((0, T) \times \Omega ; \mathbb{R}^{3}\right) \cap L^{\infty}\left(0, T ; W^{1,2}\left(\Omega ; \mathbb{R}^{3}\right)\right) \cap L^{2}\left(0, T ; W^{2,2}\left(\Omega ; \mathbb{R}^{3}\right)\right), \\
\vartheta \in L^{\infty}\left(0, T ; L^{1}(\Omega)\right) \cap L^{p}\left(0, T ; W^{1, p}(\Omega)\right), 1 \leq p<5 / 4, \vartheta>0 \text { a.e. in }(0, T) \times \Omega,
\end{gathered}
$$

with the pressure $p$,

$$
p \in L^{5 / 3}((0, T) \times \Omega) .
$$

The rest of the paper is devoted to the proof of Theorem 3.1 .

\section{A priori bounds}

We establish a number of formal a priori estimates. These will assume a rigorous character in the framework of the approximation scheme presented in Section 5 below.

Combining (2.19) (multiplied by a positive constant $K>0$ ) with (2.21) we obtain the total dissipation balance in the form

$$
\int_{\Omega}\left(\frac{K}{2}|\mathbf{u}|^{2}+(K \vartheta-\Lambda(\vartheta))+\frac{\left|\nabla_{x} \mathbf{d}\right|^{2}}{2}+W(\mathbf{d})\right)(\tau, \cdot)
$$




$$
\begin{gathered}
+\int_{0}^{\tau} \int_{\Omega}\left(|\Delta \mathbf{d}-\partial W(\mathbf{d})|^{2}+\frac{1}{\lambda(\vartheta)} \mathbb{S}: \nabla_{x} \mathbf{u}-\mathbf{q} \cdot \nabla_{x} \vartheta \frac{\lambda^{\prime}(\vartheta)}{\lambda^{2}(\vartheta)}\right) \\
\quad \leq \int_{\Omega}\left(\frac{K}{2}\left|\mathbf{u}_{0}\right|^{2}+\left(K \vartheta_{0}-\Lambda\left(\vartheta_{0}\right)\right)+\frac{\left|\nabla_{x} \mathbf{d}_{0}\right|^{2}}{2}+W\left(\mathbf{d}_{0}\right)\right) .
\end{gathered}
$$

For $K$ sufficiently large, the terms on the left hand side in (4.1) turn out to be nonnegative, and, in accordance with hypothesis (3.14), the integral on the right-hand side is bounded; hence we deduce the a priori bounds

$$
\begin{gathered}
\mathbf{u} \in L^{\infty}\left(0, T ; L^{2}\left(\Omega ; \mathbb{R}^{3}\right)\right) \cap L^{2}\left(0, T ; W^{1,2}\left(\Omega ; \mathbb{R}^{3}\right)\right) \cap L^{10 / 3}\left((0, T) \times \Omega ; \mathbb{R}^{3}\right), \\
\vartheta, \log (\vartheta) \in L^{\infty}\left(0, T ; L^{1}(\Omega)\right) \\
\mathbf{d} \in L^{\infty}\left(0, T ; W^{1,2}\left(\Omega ; \mathbb{R}^{3}\right)\right)
\end{gathered}
$$

where we have used (3.11-3.13).

The next step is to take the scalar product of equation (3.8) with $\mathbf{d}$ yielding

$$
\partial_{t}|\mathbf{d}|^{2}+\mathbf{u} \cdot \nabla_{x}|\mathbf{d}|^{2}+2 \partial W(\mathbf{d}) \cdot \mathbf{d}=\Delta|\mathbf{d}|^{2}-2\left|\nabla_{x} \mathbf{d}\right|^{2} .
$$

By virtue of hypothesis (3.11), we may apply the standard maximum principle to $|\mathbf{d}|^{2}$ to obtain

$$
\mathbf{d} \in L^{\infty}\left((0, T) \times \Omega ; \mathbb{R}^{3}\right) .
$$

Now, going back to (4.1) and making use of (4.6), we get

$$
\mathbf{d} \in L^{2}\left(0, T ; W^{2,2}\left(\Omega ; \mathbb{R}^{3}\right)\right),
$$

which, together with Gagliardo-Nirenberg interpolation inequality (cf. [14, p. 125])

$$
\|\nabla \boldsymbol{d}\|_{L^{4}(\Omega)} \leq c_{1}\|\Delta \boldsymbol{d}\|_{L^{2}(\Omega)}^{1 / 2}\|\boldsymbol{d}\|_{L^{\infty}(\Omega)}^{1 / 2}+c_{2}\|\boldsymbol{d}\|_{L^{\infty}(\Omega)}
$$

gives rise to

$$
\nabla_{x} \mathbf{d} \in L^{4}((0, T) \times \Omega) .
$$

This estimate turns out to be "crucial" in order to obtain a bound for the pressure and, in general, for the proof of existence of solutions.

Thanks to our choice of the slip boundary conditions (2.15) for the velocity, the pressure $p$ can be "computed" directly from (3.8) as the unique solution of the elliptic problem

$$
\Delta p=\operatorname{div} \operatorname{div}\left(\mathbb{S}-\lambda(\vartheta) \nabla_{x} \mathbf{d} \odot \nabla_{x} \mathbf{d}-\mathbf{u} \otimes \mathbf{u}\right),
$$

supplemented with the boundary condition

$$
\partial_{\mathbf{n}} p=\left(\operatorname{div}\left(\mathbb{S}-\lambda(\vartheta) \nabla_{x} \mathbf{d} \odot \nabla_{x} \mathbf{d}-\mathbf{u} \otimes \mathbf{u}\right)\right) \cdot \mathbf{n} \text { on } \partial \Omega .
$$

To be precise, the last two relations have to be interpreted in a "very weak" sense. Namely, the pressure $p$ is determined through a family of integral identities

$$
\int_{\Omega} p \Delta \varphi=\int_{\Omega}\left(\mathbb{S}-\lambda(\vartheta) \nabla_{x} \mathbf{d} \odot \nabla_{x} \mathbf{d}-\mathbf{u} \otimes \mathbf{u}\right): \nabla_{x}^{2} \varphi
$$


for any test function $\varphi \in C^{\infty}(\bar{\Omega}),\left.\nabla_{x} \varphi \cdot \mathbf{n}\right|_{\partial \Omega}=0$. Consequently, the bounds established in (4.2), (4.9) may be used, together with the standard elliptic regularity results, to conclude that

$$
p \in L^{5 / 3}((0, T) \times \Omega) .
$$

Finally, we turn attention to the heat equation (2.13). Multiplying (2.13) by $H^{\prime}(\vartheta)$ (for a generic $\left.H \in C^{2}([0,+\infty))\right)$ we deduce its "renormalized" form

$$
\begin{gathered}
\partial_{t} H(\vartheta)+\operatorname{div}(H(\vartheta) \mathbf{u})+\operatorname{div}\left(H^{\prime}(\vartheta) \mathbf{q}\right) \\
+H^{\prime \prime}(\vartheta)\left(\kappa(\vartheta)\left|\nabla_{x} \vartheta\right|^{2}+\left(\kappa_{\|}-\kappa_{\perp}\right)(\vartheta)\left|\mathbf{d} \cdot \nabla_{x} \vartheta\right|^{2}\right) \\
=H^{\prime}(\vartheta)\left(\mathbb{S}-\lambda(\vartheta) \nabla_{x} \mathbf{d} \odot \nabla_{x} \mathbf{d}\right): \nabla_{x} \mathbf{u} \quad \text { in } \mathcal{D}^{\prime}((0, T) \times \Omega) .
\end{gathered}
$$

The choice $H(\vartheta)=(1+\vartheta)^{\eta}, \eta<1$, in (4.12), together with the uniform bounds obtained in (4.2), (4.3), and (4.9), yield

$$
\nabla_{x}(1+\vartheta)^{\nu} \in L^{2}\left((0, T) \times \Omega ; \mathbb{R}^{3}\right) \text { for any } 0<\nu<\frac{1}{2} .
$$

Now, we apply an interpolation argument already exploited in [2. Using (4.3) and (4.13) and interpolating between $\vartheta \in L^{\infty}\left(0, T ; L^{1}(\Omega)\right)$ and $\vartheta^{\nu} \in L^{1}\left(0, T ; L^{3}(\Omega)\right)$, for $\nu \in(0,1]$, we immediately get

$$
\vartheta \in L^{q}((0, T) \times \Omega) \text { for any } 1 \leq q<5 / 3 .
$$

Further, observing that, for all $p \in[1,5 / 4)$ and $\nu>0$,

$$
\int_{(0, T) \times \Omega}|\nabla \vartheta|^{p} \leq\left(\int_{(0, T) \times \Omega}|\nabla \vartheta|^{2} \vartheta^{\nu-1}\right)^{\frac{p}{2}}\left(\int_{(0, T) \times \Omega} \vartheta^{(1-\nu) \frac{p}{2-p}}\right)^{\frac{2-p}{2}}
$$

we conclude from (4.13) and (4.14) that

$$
\nabla_{x} \vartheta \in L^{p}\left((0, T) \times \Omega ; \mathbb{R}^{3}\right) \text { for any } 1 \leq p<5 / 4 .
$$

The a priori estimates derived in this section coincide with the regularity class (3.15 3.18). Moreover, it can be shown that the solution set of (3.6 3.9) is weakly stable (compact) with respect to these bounds, namely, any sequence of (weak) solutions that complies with uniform bounds established above has a subsequence that converges to some limit. Leaving the proof of weak sequential stability to the interested reader, we pass directly to the proof of Theorem 3.1 constructing a suitable family of approximate problems whose solutions weakly converges (up to subsequences) to limit functions which solve the problem in the weak sense specified in Subsection 3.1 .

\section{Approximations}

Solutions to the Navier-Stokes system (3.6), (3.7) will be constructed by means of the nowadays standard Faedo-Galerkin approximation scheme, see Temam [16]. Let 
$W_{n, \sigma}^{1,2}\left(\Omega ; \mathbb{R}^{3}\right)$ be the Sobolev space of solenoidal functions satisfying the impermeability boundary condition, specifically,

$$
W_{n, \sigma}^{1,2}=\left\{\mathbf{v} \in W^{1,2}\left(\Omega ; \mathbb{R}^{3}\right) \mid \operatorname{div} \mathbf{v}=0 \text { a.e. in } \Omega,\left.\mathbf{v} \cdot \mathbf{n}\right|_{\partial \Omega}=0\right\} .
$$

Since $\partial \Omega$ is of class $C^{2+\nu}$, there exists an orthonormal basis $\left\{\mathbf{v}_{n}\right\}_{n=1}^{\infty}$ of the Hilbert space $W_{n, \sigma}^{1,2}$ such that $\mathbf{v}_{n} \in C^{2+\nu}$, see [6, Theorem 10.13]. We take $M \leq N$ and denote $X_{N}=\operatorname{span}\left\{\mathbf{v}_{n}\right\}_{n=1}^{N}$. Our strategy is to pass to the limit first for $N \rightarrow \infty$ and then for $M \rightarrow \infty$.

The aproximate velocity fields $\mathbf{u}_{N, M} \in C^{1}\left([0, T] ; X_{N}\right)$ solve the Faedo-Galerkin system

$$
\begin{gathered}
\frac{\mathrm{d}}{\mathrm{d} t} \int_{\Omega} \mathbf{u}_{N, M} \cdot \mathbf{v}=\int_{\Omega} \mathbf{u}_{N, M} \otimes\left[\mathbf{u}_{N, M}\right]_{M}: \nabla_{x} \mathbf{v} \\
-\int_{\Omega} \mu\left(\vartheta_{N, M}\right)\left(\nabla_{x} \mathbf{u}_{N, M}+\nabla_{x}^{t} \mathbf{u}_{N, M}\right): \nabla_{x} \mathbf{v}+\int_{\Omega} \lambda\left(\vartheta_{N, M}\right) \nabla_{x} \mathbf{d}_{N, M} \odot \nabla_{x} \mathbf{d}_{N, M}: \nabla_{x} \mathbf{v} \\
\int_{\Omega} \mathbf{u}_{N, M}(0, \cdot) \cdot \mathbf{v}=\int_{\Omega} \mathbf{u}_{0} \cdot \mathbf{v}
\end{gathered}
$$

for any $\mathbf{v} \in X_{N}$. Here, the symbol $[\mathbf{v}]_{M}$ denotes the orthogonal projection onto the space $\operatorname{span}\left\{V_{n}\right\}_{n=1}^{M}$.

The functions $\mathbf{d}_{N, M}$ are determined in terms of $\mathbf{u}_{N, M}$ as the unique solution of the parabolic system

$$
\partial_{t} \mathbf{d}_{N, M}+\left[\mathbf{u}_{N, M}\right]_{M} \cdot \nabla_{x} \mathbf{d}_{N, M}+\partial W\left(\mathbf{d}_{N, M}\right)=\Delta \mathbf{d}_{N, M},
$$

supplemented with

$$
\begin{gathered}
\left.\nabla_{x}\left(d_{N, M}\right)_{i} \cdot \mathbf{n}\right|_{\partial \Omega}=0, i=1,2,3, \\
\mathbf{d}_{N, M}(0, \cdot)=\mathbf{d}_{0, M},
\end{gathered}
$$

where $\mathbf{d}_{0, M}$ is a suitable smooth approximation of $\mathbf{d}_{0}$.

Next, given $\mathbf{u}_{N, M}, \mathbf{d}_{N, M}$, the temperature $\vartheta_{N, M}$ is evaluated my means of the heat equation (cf. Ladyženskaja et al. [8, Chapter V, Theorem 8.1])

$$
\begin{aligned}
& \partial_{t} \vartheta_{N, M}+\operatorname{div}\left(\vartheta_{N, M} \mathbf{u}_{N, M}\right)+\operatorname{div} \mathbf{q}_{N, M}= \mathbb{S}_{N, M}: \nabla_{x} \mathbf{u}_{N, M} \\
&-\left(\lambda\left(\vartheta_{N, M}\right)\left(\nabla_{x} \mathbf{d}_{N, M} \odot \nabla_{x} \mathbf{d}_{N, M}\right)\right): \nabla_{x} \mathbf{u}_{N, M}, \\
&\left.\mathbf{q}_{N, M} \cdot \mathbf{n}\right|_{\partial \Omega}=0, \\
& \vartheta_{N, M}(0, \cdot)=\vartheta_{0, M},
\end{aligned}
$$

where $\mathbb{S}_{N, M}=\mu\left(\vartheta_{N, M}\right)\left(\nabla_{x} \mathbf{u}_{N, M}+\nabla_{x}^{t} \mathbf{u}_{N, M}\right)$, and

$$
\mathbf{q}_{N, M}=-\kappa\left(\vartheta_{N, M}\right) \nabla_{x} \vartheta_{N, M}-\left(\kappa_{\|}-\kappa_{\perp}\right)\left(\vartheta_{N, M}\right) \mathbf{d}_{N, M}\left(\mathbf{d}_{N, M} \cdot \nabla_{x} \vartheta_{N, M}\right) .
$$

Finally, the pressure $p_{N, M}$ is determined as the unique solution of a system of integral identities

$$
\int_{\Omega} p_{N, M} \Delta \varphi=\int_{\Omega}\left(\mathbb{S}_{N, M}-\lambda\left(\vartheta_{N, M}\right) \nabla_{x} \mathbf{d}_{N, M} \odot \nabla_{x} \mathbf{d}_{N, M}-\mathbf{u}_{N, M} \otimes\left[\mathbf{u}_{N, M}\right]_{M}\right): \nabla_{x}^{2} \varphi
$$


satisfied for any test function $\varphi \in C^{\infty}(\bar{\Omega}),\left.\nabla_{x} \varphi \cdot \mathbf{n}\right|_{\partial \Omega}=0$. In particular, we immediately deduce the estimate

$$
\left\|p_{N, M}\right\|_{L^{2}((0, T) \times \Omega)} \leq C(M) .
$$

Now, taking $H(\vartheta)=(1+\vartheta)^{\nu}$, with $\nu \in(0,1 / 2)$, in (4.12), we get

$$
\left\|\partial_{t} \vartheta_{N, M}^{\nu}\right\|_{\left(C^{0}\left([0, T] ; W^{1, r}(\Omega)\right)\right)^{*}} \leq C\left\|\partial_{t} \vartheta_{N, M}^{\nu}\right\|_{L^{1}((0, T) \times \Omega)} \leq C,
$$

where $C$ is a positive constant independent of $N$ and $M$, with $r \in(3,+\infty), \nu \in$ $(0,1 / 2)$.

Regularizing the convective terms in (5.1), (5.2) is in the spirit of Leray's original approach [9] to the Navier-Stokes system. As a result, we recover the internal energy equality at the level of the limit $N \rightarrow \infty$. This fact, in turn, enables us to replace the internal energy equation (5.5) by the total energy balance before performing the limit $M \rightarrow \infty$. For fixed $M, N$, problem (5.1 - 5.8) can be solved by means of a simple fixed point argument, exactly as in [6, Chapter 3]. Note that all a priori bounds derived formally in Section 4 apply to our approximate problem. Thus given $\mathbf{u} \in C\left([0, T] ; X_{N}\right)$, we find $\mathbf{d}=\mathbf{d}[\mathbf{u}]$ solving (5.2 - 5.4), and then $\vartheta=\vartheta[\mathbf{u}, \mathbf{d}]$ and the pressure $p$ satisfying (5.5-5.8). Plugging these $\mathbf{d}, \vartheta$ in (5.1) we may find a new function $\mathcal{T}[\mathbf{u}]$ defining thus a mapping $\mathbf{u} \mapsto \mathcal{T}[\mathbf{u}]$. Given the a priori bounds obtained in Section 4 , we can easily show that $\mathcal{T}$ possesses a fixed point by means of the classical Schauder's argument, at least on a possibly short time interval. However, using once more the a priori estimates we easily conclude that the approximate solutions can be extended on any fixed time interval $[0, T]$ (see [6, Chapter 6] for details).

\subsection{Passage to the limit as $N \rightarrow \infty$}

Having established the existence of the approximate solutions $\mathbf{u}_{N, M}, \mathbf{d}_{N, M}, \vartheta_{N, M}$, and $p_{N, M}$, we let $N \rightarrow \infty$ and use the uniform bounds established in Section 4 to obtain

$$
\begin{aligned}
& \mathbf{u}_{N, M} \rightarrow \mathbf{u}_{M} \text { weakly-(*) in } L^{\infty}\left(0, T ; L^{2}\left(\Omega ; \mathbb{R}^{3}\right)\right) \cap L^{2}\left(0, T ; W^{1,2}\left(\Omega ; \mathbb{R}^{3}\right)\right), \\
& \partial_{t} \mathbf{u}_{N, M} \rightarrow \partial_{t} \mathbf{u}_{M} \text { weakly in } L^{2}\left(0, T ;\left(W^{1,2}\left(\Omega ; \mathbb{R}^{3}\right)\right)^{*}\right), \\
& p_{N, M} \rightarrow p_{M} \text { weakly in } L^{2}((0, T) \times \Omega), \\
& \vartheta_{N, M}^{\nu} \rightarrow \vartheta_{M}^{\nu} \quad \text { weakly-(*) in } L^{2}\left(0, T ; W^{1,2}(\Omega)\right) \cap L^{\infty}\left(0, T ; L^{1 / \nu}(\Omega)\right), \\
& \partial_{t} \vartheta_{N, M}^{\nu} \rightarrow \partial_{t} \vartheta_{M}^{\nu} \quad \text { weakly-(*) in }\left(C_{0}\left(0, T ; W^{1, r}(\Omega)\right)\right)^{*}, \\
& \boldsymbol{d}_{N, M} \rightarrow \boldsymbol{d}_{M} \text { weakly-(*) in } L^{\infty}\left(0, T ; W^{1,2}\left(\Omega ; \mathbb{R}^{3}\right)\right) \cap L^{4}\left(0, T ; W^{2,4}\left(\Omega ; \mathbb{R}^{3}\right)\right), \\
& \partial_{t} \boldsymbol{d}_{N, M} \rightarrow \partial_{t} \boldsymbol{d}_{M} \text { weakly in } L^{4}\left(0, T ; L^{4}\left(\Omega ; \mathbb{R}^{3}\right)\right),
\end{aligned}
$$

for any $\nu \in(0,1 / 2)$, and $r>3$. Note that at this stage $M$ remains fixed in the convective term $\mathbf{u}_{N, M} \otimes\left[\mathbf{u}_{N, M}\right]_{M}$.

Hence, applying the Aubin-Lions compactness lemma (cf. [15]), we deduce that

$$
\begin{aligned}
& \mathbf{u}_{N, M} \rightarrow \mathbf{u}_{M} \text { strongly in } L^{2}\left(0, T ; L^{2}\left(\Omega ; \mathbb{R}^{3}\right)\right), \\
& \vartheta_{N, M} \rightarrow \vartheta \text { strongly in } L^{p}((0, T) \times \Omega)
\end{aligned}
$$


for any $p \in[1,5 / 3)$. Moreover, at this level of approximation, the director field $\mathbf{d}_{M}$ is regular, and, in particular, we have

$$
\nabla_{x} \boldsymbol{d}_{N, M} \rightarrow \nabla_{x} \boldsymbol{d}_{M} \text { strongly in } L^{4}((0, T) \times \Omega) .
$$

Hence, we can perform the limit passage

$$
\begin{gathered}
\lambda\left(\vartheta_{N, M}\right)\left(\nabla_{x} \mathbf{d}_{N, M} \odot \nabla_{x} \mathbf{d}_{N, M}\right): \nabla_{x} \mathbf{u}_{N, M} \\
\rightarrow \lambda\left(\vartheta_{M}\right)\left(\nabla_{x} \mathbf{d}_{M} \odot \nabla_{x} \mathbf{d}_{M}\right): \nabla_{x} \mathbf{u}_{M} \text { in, say, } L^{1}((0, T) \times \Omega) .
\end{gathered}
$$
problem

Thus we may infer that the limit quantities $\mathbf{u}_{M}, \mathbf{d}_{M}, \vartheta_{M}$, and $p_{M}$ solve the

$$
\int_{\Omega} \mathbf{u}_{M}(t, \cdot) \cdot \nabla_{x} \varphi=0 \text { for a.a. } t \in(0, T)
$$

for any test function $\varphi \in C^{\infty}(\bar{\Omega})$,

$$
\int_{0}^{T} \int_{\Omega}\left(\mathbf{u}_{M} \cdot \partial_{t} \varphi+\mathbf{u}_{M} \otimes\left[\mathbf{u}_{M}\right]_{M}: \nabla_{x} \varphi\right)=\int_{0}^{T} \int_{\Omega} \mathbb{T}_{M}: \nabla_{x} \varphi-\int_{\Omega} \mathbf{u}_{0} \cdot \varphi(0, \cdot)
$$

for any $\varphi \in C_{0}^{\infty}\left([0, T) \times \bar{\Omega} ; \mathbb{R}^{3}\right),\left.\varphi \cdot \mathbf{n}\right|_{\partial \Omega}=0$,

$$
\partial_{t} \mathbf{d}_{M}+\left[\mathbf{u}_{M}\right]_{M} \cdot \nabla_{x} \mathbf{d}_{M}+\partial W\left(\mathbf{d}_{M}\right)=\Delta \mathbf{d}_{M}, \quad \text { a.e. in }(0, T) \times \Omega,
$$

supplemented with

$$
\begin{aligned}
\left.\nabla_{x}\left(d_{M}\right)_{i} \cdot \mathbf{n}\right|_{\partial \Omega} & =0, i=1,2,3 \\
\mathbf{d}_{M}(0, \cdot) & =\mathbf{d}_{0, M},
\end{aligned}
$$

and

$$
\partial_{t} \vartheta_{M}+\operatorname{div}\left(\vartheta_{M} \mathbf{u}_{M}\right)+\operatorname{div} \mathbf{q}_{M} \geq \mathbb{S}_{M}: \nabla_{x} \mathbf{u}_{M}-\lambda\left(\vartheta_{M}\right)\left(\nabla_{x} \mathbf{d}_{M} \odot \nabla_{x} \mathbf{d}_{M}\right): \nabla_{x} \mathbf{u}_{M}
$$

in the sense of distributions with non-negative test functions,

$$
\begin{gathered}
\left.\mathbf{q}_{M} \cdot \mathbf{n}\right|_{\partial \Omega}=0, \\
\vartheta_{M}(0, \cdot)=\vartheta_{0, M},
\end{gathered}
$$

together with the total energy balance

$$
\frac{\mathrm{d}}{\mathrm{d} t} \int_{\Omega}\left(\frac{1}{2}\left|\mathbf{u}_{M}\right|^{2}+\vartheta_{M}\right)=0
$$

where

$$
\mathbb{T}_{M}=\mathbb{S}_{M}-\lambda\left(\vartheta_{M}\right)\left(\nabla_{x} \boldsymbol{d}_{M} \odot \nabla_{x} \boldsymbol{d}_{M}\right)-p_{M} \mathbb{I},
$$

and

$$
\mathbb{S}_{M}=\mu\left(\vartheta_{M}\right)\left(\nabla_{x} \mathbf{u}_{M}+\nabla_{x}^{t} \mathbf{u}_{M}\right)
$$


Moreover, since the convective term $\mathbf{u}_{M} \otimes\left[\mathbf{u}_{M}\right]_{M}$ is regular (and consequently $\left.\partial_{t} \mathbf{u}_{M} \in L^{2}\left(0, T ;\left(W^{1,2}\left(\Omega ; \mathbb{R}^{3}\right)\right)^{*}\right)\right)$, we can take $\mathbf{u}_{M}$ as a test function in (5.19) to recover the kinetic energy balance in the form

$$
\begin{aligned}
\left\|\mathbf{u}_{M}(t)\right\|_{L^{2}(\Omega)}^{2} & +\int_{0}^{t} \int_{\Omega} \mu\left(\vartheta_{M}\right)\left|\nabla_{x} \mathbf{u}_{M}+\nabla_{x}^{t} \mathbf{u}_{M}\right|^{2}=\left\|\mathbf{u}_{0}\right\|_{L^{2}(\Omega)}^{2} \\
& +2 \int_{0}^{t} \int_{\Omega} \lambda\left(\vartheta_{M}\right)\left(\nabla_{x} \mathbf{d}_{M} \odot \nabla_{x} \mathbf{d}_{M}\right): \nabla_{x} \mathbf{u}_{M}
\end{aligned}
$$

Similarly, taking $\mathbf{v}=\mathbf{u}_{N, M}$ in (5.1) we get

$$
\begin{aligned}
\left\|\mathbf{u}_{N, M}(t)\right\|_{L^{2}(\Omega)}^{2} & +\int_{0}^{t} \int_{\Omega} \mu\left(\vartheta_{M, N}\right)\left|\nabla_{x} \mathbf{u}_{N, M}+\nabla_{x}^{t} \mathbf{u}_{N, M}\right|^{2}=\left\|\mathbf{u}_{0}\right\|_{L^{2}(\Omega)}^{2} \\
& +2 \int_{0}^{t} \int_{\Omega} \lambda\left(\vartheta_{N, M}\right)\left(\nabla_{x} \mathbf{d}_{N, M} \odot \nabla_{x} \mathbf{d}_{N, M}\right): \nabla_{x} \mathbf{u}_{N, M} .
\end{aligned}
$$

Passing to the limit $N \rightarrow \infty$ in the last equation, using (5.16) and (3.13), and comparing the result with (5.29), we conclude, by means of (3.12), that

$$
\nabla_{x} \mathbf{u}_{N, M} \rightarrow \nabla_{x} \mathbf{u}_{M} \text { strongly in } L^{2}((0, T) \times \Omega) .
$$

Accordingly, the inequality (5.23) may be replaced by

$$
\partial_{t} \vartheta_{M}+\operatorname{div}\left(\vartheta_{M} \mathbf{u}_{M}\right)+\operatorname{div} \mathbf{q}_{M}=\mathbb{S}_{M}: \nabla_{x} \mathbf{u}_{M}-\lambda\left(\vartheta_{M}\right)\left(\nabla_{x} \mathbf{d}_{M} \odot \nabla_{x} \mathbf{d}_{M}\right): \nabla_{x} \mathbf{u}_{M}
$$

As a matter of fact, (5.31) already follows from (5.23), (5.26), and (5.29).

Moreover, taking in (5.19) $\mathbf{u}_{M} \varphi$ (with $\varphi \in \mathcal{D}((0, T) \times \Omega)$ ) in place of $\varphi$, we get

$$
\begin{array}{r}
\partial_{t}\left(\frac{1}{2}\left|\mathbf{u}_{M}\right|^{2}+\vartheta_{M}\right)+\operatorname{div}\left(\left(\frac{1}{2}\left|\mathbf{u}_{M}\right|^{2}+\vartheta_{M}\right)\left[\mathbf{u}_{M}\right]_{M}\right)+\operatorname{div}\left(p_{M} \mathbf{u}_{M}\right)+\operatorname{div} \mathbf{q}_{M} \\
=\operatorname{div}\left(\mathbb{S}_{M} \mathbf{u}_{M}\right)-\operatorname{div}\left(\lambda\left(\vartheta_{M}\right)\left(\nabla_{x} \boldsymbol{d}_{M} \odot \nabla_{x} \boldsymbol{d}_{M}\right) \mathbf{u}_{M}\right) \operatorname{in} \mathcal{D}^{\prime}((0, T) \times \Omega) .
\end{array}
$$

This concludes the passage to the limit for $N \rightarrow \infty$.

\subsection{Passage to the limit as $M \rightarrow \infty$}

Our final goal is to let $M \rightarrow \infty$ (5.19 5.26). We notice that the limits in (5.9), (5.12 5.13), (5.16 5.17) still hold when letting $M \rightarrow \infty$. Moreover, we have

$$
\begin{aligned}
& \partial_{t} \mathbf{u}_{M} \rightarrow \partial_{t} \mathbf{u} \text { weakly in } L^{5 / 3}\left(0, T ; W^{-1,5 / 3}\left(\Omega ; \mathbb{R}^{3}\right)\right) \\
& p_{M} \rightarrow p \text { weakly in } L^{5 / 3}((0, T) \times \Omega) \\
& \boldsymbol{d}_{M} \rightarrow \boldsymbol{d} \text { weakly-(*) in } L^{\infty}\left((0, T) \times \Omega ; \mathbb{R}^{3}\right) \cap L^{\infty}\left(0, T ; W^{1,2}\left(\Omega ; \mathbb{R}^{3}\right)\right) \\
& \qquad L^{2}\left(0, T ; W^{2,2}\left(\Omega ; \mathbb{R}^{3}\right)\right), \\
& \partial_{t} \boldsymbol{d}_{M} \rightarrow \partial_{t} \boldsymbol{d} \text { weakly in } L^{2}\left(0, T ; L^{3 / 2}\left(\Omega ; \mathbb{R}^{3}\right)\right) .
\end{aligned}
$$


Now, we can easily pass to the limit $M \rightarrow \infty$ in (5.18 5.22) to recover (3.6 3.8). In addition, by virtue of (4.2), (4.9), (4.14), we get

$$
\begin{aligned}
& \left\{\left(\frac{\left|\mathbf{u}_{M}\right|^{2}}{2}+p_{M}\right)\left[\mathbf{u}_{M}\right]_{M}\right\}_{M>0} \text { bounded in } L^{10 / 9}((0, T) \times \Omega), \\
& \left\{\vartheta_{M} \mathbf{u}_{M}\right\}_{M>0} \text { bounded in } L^{q}\left(0, T ; L^{q}(\Omega)\right) \text { for any } q \in[1,10 / 9), \\
& \left\{\mathbb{S}_{M} \mathbf{u}_{M}\right\}_{M>0} \text { bounded in } L^{5 / 4}((0, T) \times \Omega), \\
& \left\{\lambda\left(\vartheta_{M}\right)\left(\nabla_{x} \boldsymbol{d}_{M} \odot \nabla_{x} \boldsymbol{d}_{M}\right) \mathbf{u}_{M}\right\}_{M>0} \text { bounded in } L^{5 / 4}\left(0, T ; L^{5 / 4}(\Omega)\right) .
\end{aligned}
$$

Consequently, we can pass to the limit in (5.32) to deduce the desired conclusion (3.9).

Finally, as convex functionals are weakly lower semicontinuous, we can see that (5.23) gives rise to

$$
\partial_{t} \vartheta+\operatorname{div}(\vartheta \mathbf{u})+\operatorname{div} \mathbf{q} \geq \mathbb{S}: \nabla_{x} \mathbf{u}-\lambda(\vartheta)\left(\nabla_{x} \mathbf{d} \odot \nabla_{x} \mathbf{d}\right): \nabla_{x} \mathbf{u} \text { in } \mathcal{D}^{\prime}((0, T) \times \Omega) .
$$

This completes the proof of Theorem 3.1 .

\section{References}

[1] T. Blesgen, A generalization of the Navier-Stokes equations to two-phase flow, J. Phys. D Appl. Phys., 32 (1999), 1119-1123.

[2] M. Bulíček, E. Feireisl, J. Málek, A Navier-Stokes-Fourier system for incompressible fluids with temperature dependent material coefficients, Nonlinear Anal. Real World Appl., 10 (2009), 992-1015.

[3] L. Caffarelli, R. Kohn, L. Nirenberg, Partial regularity of suitable weak solutions of the Navier-Stokes equations, Comm. Pure Appl. Math., 35 (1982), 771-831.

[4] J. Ericksen, Liquid crystals with variable degree of orientation, Arch. Ration. Mech. Anal., 113 (1991), 97-120.

[5] E. Feireisl, J. Málek, On the Navier-Stokes equations with temperature-dependent transport coefficients, Differ. Equ. Nonlinear Mech. (2006) Art. ID 90616, 14 pp. (electronic).

[6] E. Feireisl, A. Novotný, Singular limits in thermodynamics of viscous fluids, Advances in Mathematical Fluid Mechanics, Birkhäuser Verlag, Basel, 2009.

[7] F. Jiang, Z. Tan, Global weak solution to the flow of liquid crystals system, Math. Math. Appl. Sci., to appear (2009), doi: 10.1002/mma.1132.

[8] O.A. Ladyženskaja, V.A. Solonnikov, N.N. Uraltseva, Linear and quasilinear equations of parabolic type, Translations of Mathematical Monographs, Vol. 23, American Mathematical Society, Providence, R.I. 1967.

[9] J. Leray, Sur le mouvement d'un liquide visqueux emplissant l'espace. (French) Acta Math., 63 (1934), 193-248. 
[10] F.M. Leslie, Some constitutive equations for liquid crystals, Arch. Rational Mech. Anal., 28 (1968), 265-283.

[11] F.-H. Lin, C. Liu, Nonparabolic dissipative systems modeling the flow of liquid crystals, Comm. Pure Appl. Math., 48 (1995), 501-537.

[12] F.-H. Lin, C. Liu, Existence of solutions for the Ericksen-Leslie system, Arch. Ration. Mech. Anal., 154 (2000), 135-156.

[13] C. Liu, J. Shen, On liquid crystal flows with free-slip boundary conditions, Discrete Contin. Dynam. Systems, 7 (2001), 307-318.

[14] L. Nirenberg, On elliptic partial differential equations, Ann. Scuola Norm. Sup. Pisa (3), 13 (1959), 115-162.

[15] J. Simon, Compact sets in the space $L^{p}(0, T ; B)$, Ann. Mat. Pura Appl. (4), 146 (1987), 65-96.

[16] R. Temam, Navier-Stokes equations. Theory and numerical analysis, AMS Chelsea Publishing, Providence, RI, 2001 